\title{
COMPARATIVE ANALYSIS OF 3D PHOTOGRAMMETRY MODELING SOFTWARE PACKAGES FOR DRONES SURVEY
}

\author{
L. Hinge ${ }^{1}$, J. Gundorph ${ }^{1}$, U. Ujang ${ }^{1,2, *}$, S. Azri ${ }^{1,2}$, F. Anton ${ }^{3}$, and A. Abdul Rahman ${ }^{2}$ \\ ${ }^{1}$ Department of Geodesy, Denmark National Space Institute, Technical University of Denmark, Kgs. Lyngby, Denmark - (lhinge, \\ jgundorph, ujang, norsu)@ space.dtu.dk \\ ${ }^{2}$ Department of Geoinformation, Faculty of Built Environment and Survey, Universiti Teknologi Malaysia, Johor, Malaysia - \\ (mduznir, suhaibah, alias)@utm.my \\ ${ }^{3}$ Yachay Tech University, School of Mathematical Sciences and Information Technology, Ecuador - fcastro@yachaytech.edu.ec
}

Commission IV, WG IV/1

KEY WORDS: Photogrammetry, 3D Model, 3D Analysis, Drones Survey

\begin{abstract}
:
Drones are becoming popular in spatial mapping or survey. The use of drones survey can be seen from it low flying heights (capable to create a clear images), accessible on difficult or non-friendly vehicle access areas, faster data acquisition and higher data resolution henceforth improve the quality of the survey. However, this paper focuses on the post-processing of drone images for 3D surface modeling. With the motivation of producing better 3D models, four software packages are used for comparison. Those software packages are eyesMap3D, Drone Deploy, Agisoft PhotoScan and Pix4Dmapper. The equipment used to ensure a high level of quality model is the Leica GPS1200+ stationary GPS module and the DJI Phantom 4 PRO drone. The Leica GPS1200+ stationary GPS module were used to track the exact position of tie points on the ground. Meanwhile the DJI Phantom 4 PRO drone is used as data inputs (images) for the software packages stated. In addition, the drone is used to fly over a golf course, with a challenge of homogenous surface for 3D surface modeling. Based on the output, it shows that each software packages produces slightly different outputs. This paper summarizes the outputs and discusses the key elements in each software packages. This variation might be useful for future references in 3D surface modeling that can conform in different applications requirements.
\end{abstract}

\section{INTRODUCTION}

The purpose of this project is to compare different $3 \mathrm{D}$ modelling software and their capabilities in field use. Specifically it is to find the ease of modelling and precision of the software and compare these to their outputs. The data modelled was taken using a drone flying over a golf course three times. Then, by using one set of images, 3D model were created using alternatives software in order to see if there is a deviation of detail. Thus, we can deduce which software can provide the highest quality of 3D modelling. The use of drones or Unmanned Aerial Vehicle (UAV) in photogrammetry can be seen more practical in terms of time and costing. This can be seen in other research (Eisenbeiss, 2009; Nex and Remondino, 2014). However, not most cases can apply this method due to limitation of flying height, trees canopy and etc. On the other hand, this research seeks the UAV and photogrammetry outputs and it can benefited other research that requires 3D model such as (Izham et al., 2011; Suhaibah et al., 2016; Syahiirah and Uznir, 2018; Uznir et al., 2015).

\section{RESEARCH MOTIVATIONS}

\subsection{Photogrammetry}

The process and method of photogrammetry is to establish the geometric relationship between an image and an object, as it existed at the time of the imaging event. From this relationship, metric information of the object can be derived. The process of photogrammetry is closely related to, and often overlaps with the fields of Remote Sensing and Computer Vision. A photogrammetric project is classified as that which requires satellite imagery, airborne imagery and close-range or industrial applications. In aerial photography, as is the focus of this report, flight lines of the aircraft are laid out on a flight map, spaced from each other in a way that will cause the photographs to overlap, covering a common region of the ground. This is called the sidelap. In addition to this, every photograph covers an area that overlaps with the area of the previously taken photograph. The overlap percentage describes the fraction of an image that is common with the adjacent image. Forward overlap is the relationship between 2 adjacent photographs along the flight line, and the side overlap describes the relationship between 2 adjacent flight lines. Both are illustrated in the Figure 1 below:

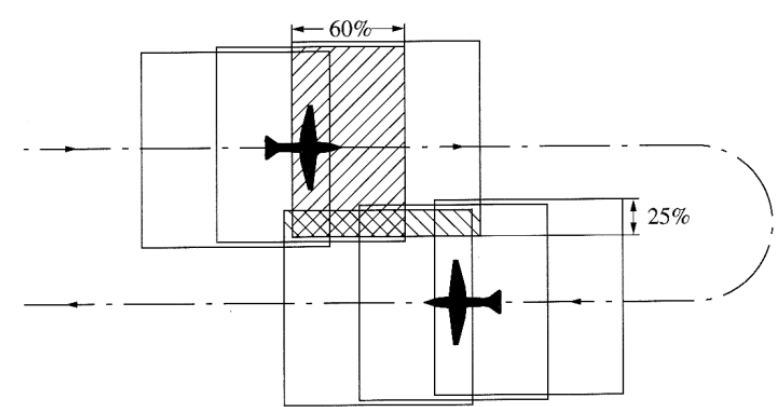

Figure 1. Aerial photography (view from above) and overlap is shown in striped (source : http://www.seos-project.eu)

These sidelaps, and especially overlaps, are very important in photogrammetry for the following reasons:

- They provide coverage of an entire ground area from several different viewpoints.

- They allow all but the central region of every photograph to be discarded, during its construction of the mosaics. 
- The small overlap area is important for constructing supplementary ground control by photogrammetric point triangulation.

The mosaics are an image product that depicts the original object. It consists of continuous pictures of the terrain constructed from many individual photographs, assembled together in a composite. It can be thought of as a series of contiguous perspective views of the ground.

\subsection{Feature Matching}

After overlapping pictures are taken, the software needs to find the 'tie points'. Tie points are points which appear in multiple, adjacent pictures. This process is known as feature matching, and is important in both photogrammetry and computer vision. There are numerous ways to do feature matching. They can be done either in the RGB or HSV spectra, where the colours are compared, or from HOG or SIFT features, where the local gradient is compared. Feature Matching is commonly performed as a three stage process, as follows:

- Extracting a number of features from the images. It is assumed here that the features are points, or lines.

- For every feature a descriptor is computed or extracted. This descriptor is used as a distinguishing representation of the feature, often as a small patch around the feature point.

- The correspondence between the two features, and thus the images, are found by a pairing of the features with their similar descriptors. Often this is done by finding the cross correlation between 2 patches, or creating histograms of the patches and calculating the distance between the 2 histograms.

\section{FLIGHT (DRONES)}

\subsection{Rules and Regulations}

There exist a great deal of rules and regulations when it comes to flying within the Danish airspace, and they are especially strict in Denmark compared to other countries. These rules exist to ensure and boost the security of the airspace, other people's rights of privacy and the general safety (Clarke, 2014; Clarke and Moses, 2014).

Different rules apply for people flying for private reasons and people flying for commercial reasons. For example, it is illegal for normal people to fly within urbanized areas, while this is allowed for people flying for a commercial reason - as long as these people have a registered drone pilot license.

In this project, a drone pilot license was used to gain greater degree of flexibility and freedom for flying with drones. This was necessary for legally flying and photographing the golf course in Hørsholm.

\subsection{Safety Zones}

When a pilot is flying with a drone, he needs to establish a safety zone (illustrated in Figure 2), which follows the drone during the entire flight. This means that the zone always has its centre at the drones' current location. The safety zone must have a radius equal to the flying height, although a minimum of $15 \mathrm{~m}$ and a maximum of $50 \mathrm{~m}$ are required.
- No other persons than the drone pilot and an eventual assistant is allowed within the safety zone.

- If the drone is fully controllable after the loss of a rotor, or has some other kind of safety measure (like a parachute) during all phases of its flight, then persons are allowed within the safety zone given that the drone pilots consent has been granted.

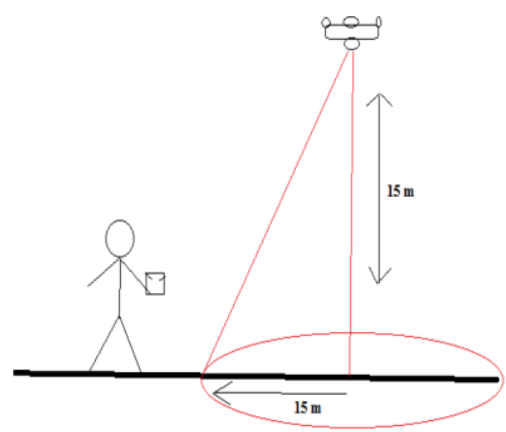

Figure 2. Illustration of drones' safety zones

Another important safety procedure to follow is ensuring that one is flying within the meteorological boundaries of the drone. This is highly individual from drone to drone, but for the DJI Phantom 4 Pro, its maximum wind speed resistance is $10 \mathrm{~m} / \mathrm{s}$. When a drone meets too high wind speeds, it will struggle to maintain its position and not be able to stabilize itself, thus draining the battery very quickly.

In some cases, the wind can overturn the drone and make it crash, or the drone will become unresponsive to the controller because it is using all its energy on stabilizing itself, instead of moving. This can be quite dangerous, as the drone pilot can suddenly lose control of the aircraft, and potentially crash into a building or even other people, either because of an overturning or because of it losing all of its battery. These rules are just few examples from the regulations of drone flying in Danish airspace.

\subsection{The Golf Course}

Drones were used in the field of many (Câmara, 2014; Chmaj and Selvaraj, 2015; Masehian and Mohamadnejad, 2015). When planning a drone flight (Figure 3), especially for the purpose of doing photogrammetry, it is very important to have optimal flying conditions. There must be enough light, so it can't be too cloudy. It mustn't rain and the wind conditions can't be too harsh. In this project, almost all of February and March, it was very hard to find a good day to fly on, which ended up postponing the whole project almost a month. So careful planning must be made when performing a drone flight.

The goal was to create the model of the golf course with an increasing amount of tie points laid out on the ground. For this purpose we had printed out paper-markers, which we would put on the golf course, so we would be able to later recognize them on the pictures. The reason for this is that in photogrammetry, it needs an overlap of the tie points between adjacent pictures. 
Often, these tie points are selected manually in the software (like EyesMap3D), but the software is also able to detect them itself in most cases. If there is no overlap between adjacent pictures, the program will not be able to perform the photogrammetry process. If just 2 pictures are missing an overlap with each other, all following pictures are useless to process with the previous ones, because the software needs a continuous overlap between every picture.

During the flight, 24 tie points were set up, lying on the golf course. Furthermore, these tie points will be used to indicate spots with GPS coordinates (measured). This too was with the thought in mind that it would make the later recognition of the selected spots easier. Unfortunately, it turned out that most software doesn't support a feature for entering Ground Control Points unless except for their business package, which this project didn't have access to. At the golf course, few golf tracks were picked beforehand. These include trees, greens, water, sandpits and/or other characteristic objects. Unfortunately, the idea had to be scraped due to interfering with guests' usage of the tracks.

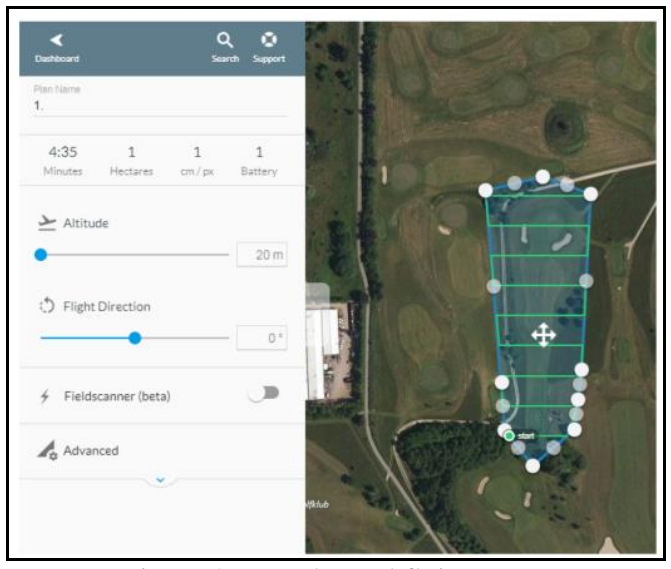

Figure 3. Pre-planned flying route

\section{3D MODELLING SOFTWARE}

Based on today's technology and updates, there are many to name tools used to model the 3D model. Previous research shows detail methods or procedures to develop the 3D model for post processing stage of drone for specific software. On contrary, this research investigates the comparison between those most used software packages, in order to produce a reference for future research. This section elaborate the software packages used in this research.

\subsection{DroneDeploy}

Drone Deploy is an online application that can both be used on a PC and a mobile device. Its main goal is to make surveying by drone easy for every kind of user. DroneDeploys services can roughly be separated into two categories:

- Flight automation and data capture - DroneDeploy allows to precisely lying out route for the drone to fly while letting users to decide when the pictures will be taken by the drone. This is helpful when the goal is to get stable and repeatable flights, with sense that the users doesn't need to manoeuvre the drone manually, thus it minimize the risk for error.
- Data processing and analysis - DroneDeploy able to generate 3D models from pictures taken by the drone. After having taken pictures of a landscape, DroneDeploy can then use those pictures to generate a 3D image of this over flown area. To do this users have to upload the pictures to their cloud. Then it will perform all the necessary computations on the dedicated servers. This saves the user a lot of computation time, and minimizes the required computation power to generate a good image/model. In this project, the first feature which is to plan the automation flight was used and then the output is compared with other software.

\subsection{EyesMap3D}

EyesMap3D allows the users to do high-density points clouds with textures achieving a realistic 3D model appearance. In addition, eyesMap3D is able to measure accurately on the images, generate true orthophotos, geo-reference and scale the results which are useful tools for customers in this software. Between the main features, the eyesMap3D's users can use their cameras, mobile phone or camera drone to capture images. Besides, eyesMap3D is compatible with most popular software packages out in the market. EyesMap3D software is capable to generate 3D models and point clouds from your photos in a short period of time. For example, you can get 2 millions of points only in 3 minutes.

\subsection{Agisoft PhotoScan}

Agisoft PhotoScan is a 3D modelling software that is capable of creating output that can be compared to the other software. It has in-built tools that let the user measure volumes and distances and it is able to plot data directly onto a map. Agisoft PhotoScan is (in contrast to DroneDeploy) processing data locally. The consequence of this is that the user needs a good computer. Agisoft PhotoScan recommends the use of a computer with at least 8GB RAM, a high speed multicore CPU $(3 \mathrm{GHz}+)$ and a GPU comparable to the Nvidia GeForce GTX 980.

\subsection{Pix4D}

Pix4D is a software developed to create georeferenced maps and models from drone imagery. Through the means of advanced photogrammetry software, it uses images to create professional orthomosaics, point clouds, models and more, purely from drone imagery. With these capabilities, PiX4D was used in this project for comparisons with other 3D modelling software.

\section{FINDINGS/RESULTS}

By using the same set of images (acquired from the drone) as an input, the 3D model was generated using the aforementioned 3D modelling software. The outputs from the software later are compared based on the visualisation of the golf course itself. 


\subsection{Visualisation - Sandbanks}

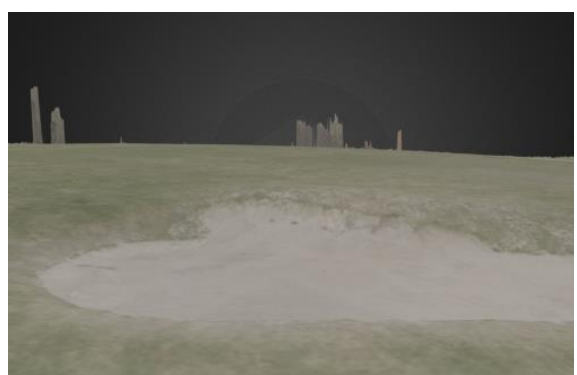

DroneDeploy

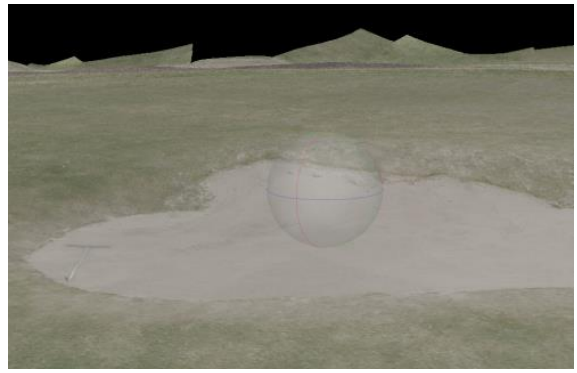

Agisoft PhotoScan

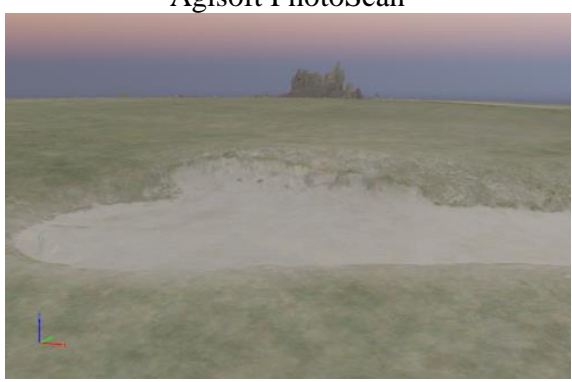

Pix4Dmapper

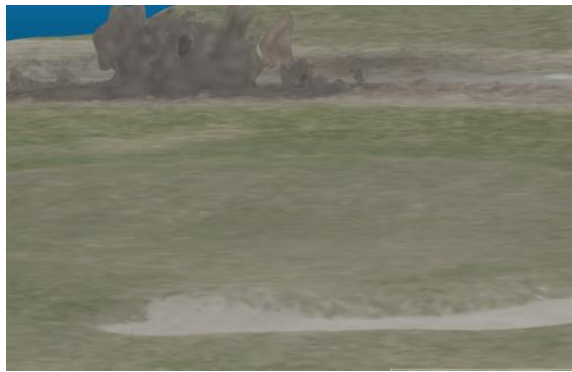

EyesMap3D

Figure 4. 3D models generated for sandbanks

Illustrated on Figure 4, DroneDeploy and Pix4Dmapper perform best at detecting the local elevations, even detecting some trees on the horizon. While EyesMap3D having a compressed detailed modeling. Agisoft PhotoScan, has the most detailed texture, but fails to show any elevations such as trees in its model. This is likely due to the fact that Agisoft PhotoScan produces a relatively low amount of points in its point cloud (400 thousand), compared to Pix4D with 4.7 million for example.

\subsection{Visualisation - Trees}

On the other hand, for trees visualisation, Figure 5 shows that Agisoft PhotoScan did not produce any trees or other objects (with distinct height), while the other three are. This is again likely due to the low-density point cloud not being able to be produced by the software. EyesMap3D produced a compressed and acceptable quality of texture and trees. Due to dense point cloud produced, Dronedeploy and Pix4D enable to shows the trees on the golf course.

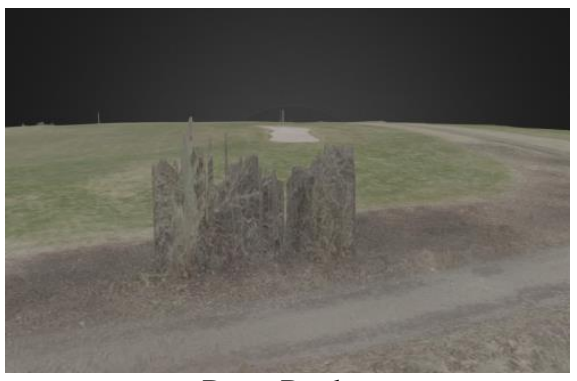

DroneDeploy

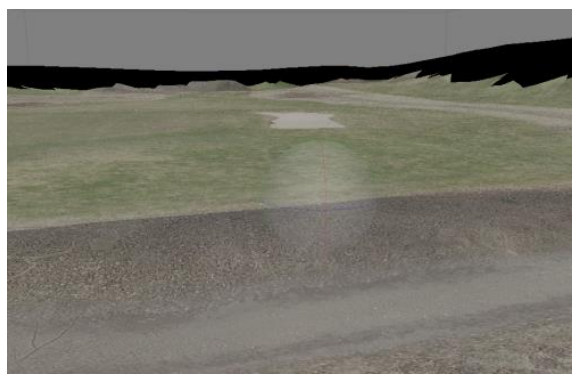

Agisoft PhotoScan

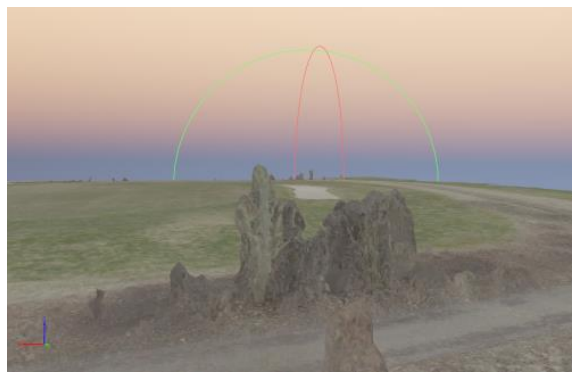

Pix4Dmapper

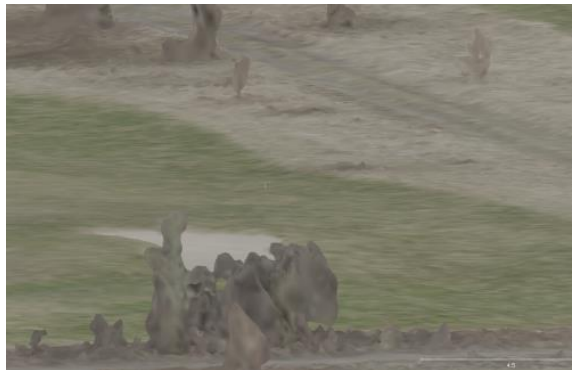

EyesMap3D

Figure 5. 3D models generated for trees

\subsection{Visualisation - Man-made Structure}

The viewpoint used for this visualisation is the place where the angle a player would look from when starting on this golf course (tee off). The green square is golf tee, the starting platform for the player. Based on the same location illustrated in Figure 6, Pix4D and EyesMap3D both have recognized the slightly slim and tall shape of the trashcan, even though they both have issues showing the exact form. This might be because 
The International Archives of the Photogrammetry, Remote Sensing and Spatial Information Sciences, Volume XLII-4/W12, 2019 5th International Conference on Geoinformation Science - GeoAdvances 2018, 10-11 October 2018, Casablanca, Morocco

its sides are made of light brown wood, which can be hard to distinguish from the brown grass on the right side. Agisoft PhotoScan has not being able to recognize any elevation while DroneDeploy is in between.

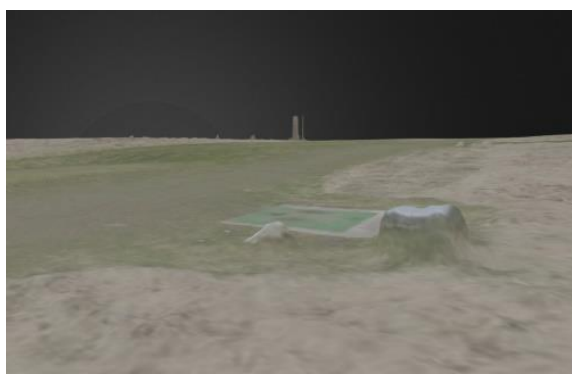

DroneDeploy

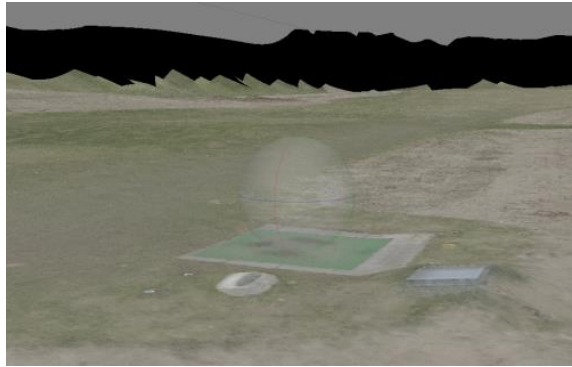

Agisoft PhotoScan

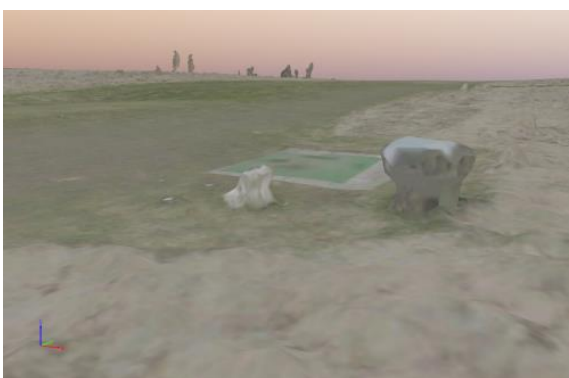

Pix4Dmapper

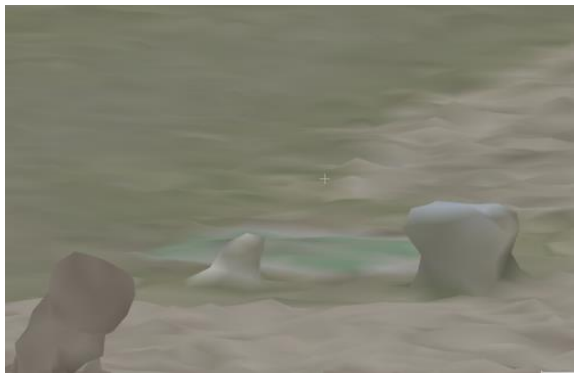

EyesMap3D

Figure 6. 3D models generated for man-made structure

\subsection{Visualisation - Sideway}

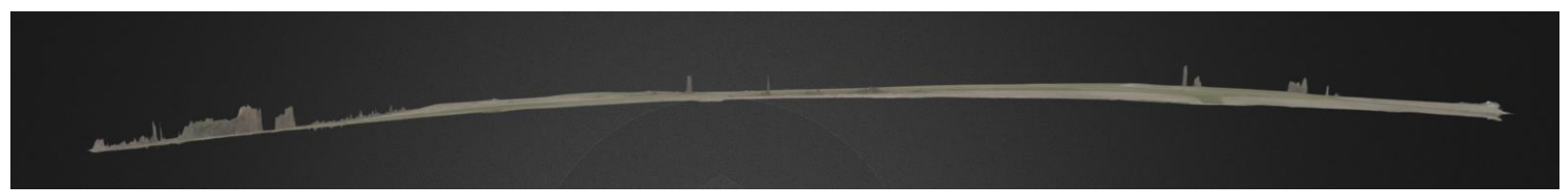

DroneDeploy

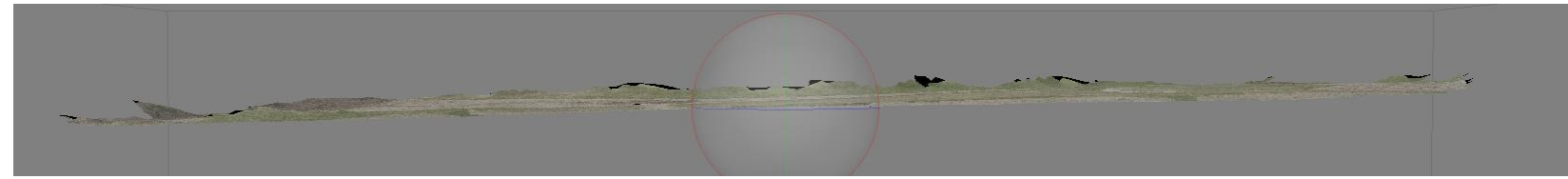

Agisoft PhotoScan

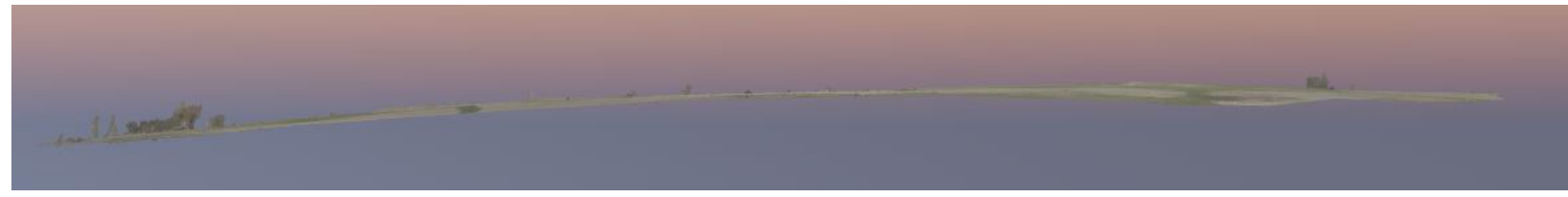

Pix4Dmapper

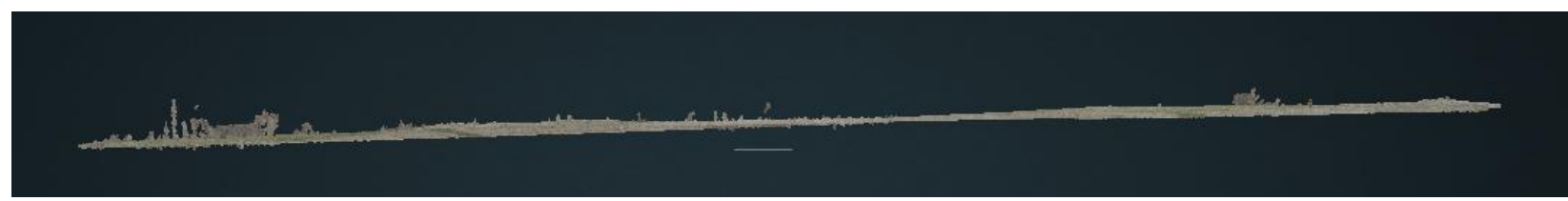

EyesMap3D

Figure 7. 3D models generated - sideways 
To purpose of visualizing the sideways is to identify which software is best at representing the actual height difference in the golf course model. By using Denmarks Height Model (DHM), the actual height difference is 5.02 meters (with an accuracy of $40 \mathrm{~cm}$ ). Based on Figure 7, DroneDeploy produced model with the strongest bend (estimate of height $22.7 \mathrm{ft}=$ $6.9 \mathrm{~m})$. Pix $4 \mathrm{D}$ is clearly the second most bent map, and it is also the one with the second highest estimate of height difference with $6 \mathrm{~m}$. The best estimate for the height difference comes from Agisoft PhotoScan. Based on the model, the height difference between the starting position and the flag is $5.2 \mathrm{~m}$, which is exactly what we found it to be in DHM. However, this research not able to find any height information from EyesMap3D.

\subsection{Source of Error}

To highlight, due to limited resource and time constraints, this research emphasis on the key points that might contribute towards the source of errors in acquiring the 3D models. The sources of errors are:

- Pictures were taken during winter, which made the trees hard to identify on the pictures (i.e. less leaves).

- Calibration error for EyesMap3D - inaccurate sensor size information due to limited resources.

- Limited ground control points or and no georeferencing conducted due to some software limitation.

- It was slightly windy on the flight day $(6-8 \mathrm{~m} / \mathrm{s})$, which could have made the drone shake a little bit, thus causing distortion in the pictures.

\section{CONCLUSION}

This research shows the output from different software packages from the aspects of visualisation for golf sandbank, trees, manmade structure and sideways. There is no the best software package that can solve the visualisation in all criteria mentioned. This research highlights which software package that best in each category. This can be seen from Pix4D and DroneDeploy that are best at modelling the local elevations like trees, benches, buildings etc. which is very important for the golf players, as they can provide the best 3D perspective of the golf course. Agisoft PhotoScan gives the by far highest resolution on their mesh. EyesMap3D can be very accurate and might even be able to provide the best 3D-modelling of all the software used. But prior knowledge in photogrammetry is required due to its method which requires camera calibration, sensor size parameter inputs and other related information. However, their new product eyesCloud3D is recommended as a better solution for a non-photogrammetry background user.

\section{ACKNOWLEDGEMENTS}

This project would give a special thanks to Hørsholm Golf club for the permission of flying over their golf course. Worth to mention, thanks to eCapture3D by giving the project permission in using their EyesMap3D product.

\section{REFERENCES}

Câmara, D., 2014. Cavalry to the rescue: Drones fleet to help rescuers operations over disasters scenarios, Antenna
Measurements \& Applications (CAMA), 2014 IEEE Conference on. IEEE, pp. 1-4.

Chmaj, G., Selvaraj, H., 2015. Distributed processing applications for UAV/drones: a survey, Progress in Systems Engineering. Springer, pp. 449-454.

Clarke, R., 2014. The regulation of civilian drones' impacts on behavioural privacy. Computer Law \& Security Review 30, 286-305.

Clarke, R., Moses, L.B., 2014. The regulation of civilian drones' impacts on public safety. Computer Law \& Security Review 30, 263-285.

Eisenbeiss, H., 2009. UAV photogrammetry. ETH Zurich.

Izham, M.Y., Uznir, U., Alias, A.R., Ayob, K., Wan Ruslan, I., 2011. Influence of georeference for saturated excess overland flow modelling using 3D volumetric soft geo-objects. Computers \& Geosciences 37, 598-609.

Masehian, E., Mohamadnejad, N., 2015. Path planning of nonholonomic flying robots using a new virtual obstacle method, Robotics and Mechatronics (ICROM), 2015 3rd RSI International Conference on. IEEE, pp. 612-617.

Nex, F., Remondino, F., 2014. UAV for 3D mapping applications: a review. Applied geomatics 6, 1-15.

Suhaibah, A., Uznir, U., Rahman, A.A., Anton, F., Mioc, D., 2016. 3D Geomarketing Segmentation: A Higher Spatial Dimension Planning Perspective. Int. Arch. Photogramm. Remote Sens. Spatial Inf. Sci. XLII-4/W1, 1-7.

Syahiirah, S., Uznir, U., 2018. Topological information extraction from buildings in CityGML. IOP Conference Series: Earth and Environmental Science 169, 012088.

Uznir, U., François, A., Ariffin, A., Darka, M., Suhaibah, A., 2015. An Amalgamation of 3D City Models in Urban Air Quality Modeling for Improving Visual Impact Analysis, in: J.W.S. Longhurst, C. Capilla, C. A. Brebbia, Barnes., J. (Eds.), Air Pollution XXIII - 23rd International Conference on Modelling, Monitoring and Management of Air Pollution. Wessex Institute, UK, València, Spain, p. 11. 